\title{
Evaluation of Thermoelectric Generators by $I-V$ Curves
}

\author{
GAO MIN, ${ }^{1,2}$ TANUJ SINGH, ${ }^{1}$ JORGE GARCIA-CANADAS, ${ }^{1}$ \\ and ROBERT ELLOR ${ }^{1}$ \\ 1.-School of Engineering, Cardiff University, Cardiff CF24 3AA, UK. 2.-e-mail: min@cf.ac.uk
}

A recent theoretical study proposes a new way to evaluate thermoelectric devices by measuring two $I-V$ curves-one obtained under a constant temperature difference and the other obtained for a constant thermal input. We report an experimental demonstration of the feasibility of this novel technique. A measurement system was designed and constructed, which enables both types of $I-V$ curves to be obtained automatically. The effective $Z T$ values of a thermoelectric module were determined using this system and compared with those measured by an impedance spectroscopy technique. The results confirm the validity of the proposed technique. In addition, the capability of measuring $Z T$ under a large temperature difference was also investigated. The results show that the $Z T$ s obtained for a large temperature difference are significantly smaller than those for a small temperature difference, providing insights into the design and operation of thermoelectric modules in realistic applications.

Key words: Thermoelectric, $I-V$ curves, maximum power output

\section{INTRODUCTION}

The performance of a thermoelectric generator is primarily evaluated by its maximum power output and conversion efficiency for given operating conditions. ${ }^{1-4}$ This is usually achieved by measuring the power output delivered to a load under a given temperature difference. Traditionally, the measurement is carried out using a variable resistor as the load. By manually changing the value of the load resistance and measuring the corresponding voltage across it at steady state, the maximum power output, together with the optimal load resistance, can be determined by plotting the power output as a function of the load resistance. A problem associated with this technique is to maintain a constant temperature difference across the thermoelectric generator when the load resistance is altered, resulting in a lengthy and inaccurate measurement. The change in the temperature difference is inevitable because of inherent thermoelectric processes described in a recent publication. ${ }^{5}$ This problem was resolved owing to advances in modern

(Received June 16, 2015; accepted October 24, 2015;

published online November 19, 2015) electronic measurement systems. Such a system uses an "electronic load" and can scan a full $I-V$ sweep within a period of less than a second, while the temperature difference across a thermoelectric generator takes a much longer time to change due to the large thermal mass and specific heat. Since the $I-V$ curves of thermoelectric devices exhibit a linear relationship, the maximum power output and corresponding optimal load resistance (also the internal resistance of thermoelectric generator) can be conveniently determined from $I-V$ curves. The use of $I-V$ curves for evaluation of thermoelectric devices is becoming a preferred method.

In addition to maximum power output and conversion efficiency, another important parameter for thermoelectric generators is the effective $Z T$, which provides insights into the fabrication quality and realistic operation performance of thermoelectric generators. The effective $Z T$ can be determined by using the Harman method. ${ }^{6}$ However, such values correspond to small temperature differences. The effective $Z T$ values under large temperature differences can be estimated by measuring the maximum temperature difference when operating in the Peltier mode ${ }^{3}$ or measuring the change in temperature difference between open-circuit and short- 
circuit when operating in the Seebeck mode. ${ }^{7}$ In this paper, we report a new method to determine the effective $Z T$ of thermoelectric generators based on $I-V$ curves. This method stems from a study of using $I-V$ curves to determine the maximum power output of a thermoelectric generator. It was observed that the slopes of $I-V$ curves are different when the scan rate is varied significantly. A theoretical work ${ }^{8}$ in an attempt to explain this observation has led to a proposal of a new method for characterisation of thermoelectric materials and devices based on two $I-V$ curves-one obtained for a constant temperature difference and the other obtained for a constant thermal input. This work focuses on the experimental validation of the proposed technique using a thermoelectric module. The effective $Z T$ is obtained from $I-V$ curves based on the principle reported in ${ }^{8}$ and compared with the values obtained using an impedance spectroscopy technique. ${ }^{9-11} \mathrm{~A}$ unique capability of this technique for measuring $Z T$ under a large temperature difference is demonstrated by comparison with the $Z T$ obtained under a small temperature difference for the same mean temperatures.

\section{PRINCIPLE OF MEASUREMENT}

Figure 1 shows a schematic diagram for measuring the $I-V$ of a thermoelectric module. It has been shown ${ }^{8}$ that, for a thermoelectric module operating under a constant temperature difference, $\Delta T_{0}$, the voltage and current relationship of the module can be expressed as,

$$
V=2 \alpha N \Delta T_{\mathrm{o}}-R_{\mathrm{i}} I
$$

where, $V$ is the voltage across the load resistance, $I$ the current flow through the circuit, $\alpha$ the Seebeck coefficient of thermoelectric materials, $N$, the number of thermocouples in a module, $R_{\mathrm{i}}$ the internal resistance of the module and $\Delta T_{0}$ the temperature difference across the module.

When the thermoelectric module is operated under a given thermal input with the initial temperature difference being $\Delta T_{\mathrm{o}}$ at open circuit condition, the voltage and current relationship is given by $^{8}$ :

$$
V=\frac{2 \alpha N \Delta T_{\mathrm{o}}}{1+Z T_{\mathrm{M}}}-R_{\mathrm{i}} I
$$

where, $Z$ is the thermoelectric figure-of-merit and $T_{\mathrm{M}}$ is a parameter related to the hot side temperature, $T_{\mathrm{H}}$, and the cold side temperature, $T_{\mathrm{C}}$, by ${ }^{8}$ :

$$
T_{\mathrm{M}}=\frac{(1+2 s) T_{\mathrm{H}}+T_{\mathrm{C}}}{2(1+s)^{2}}
$$

where, $s=R_{\mathrm{L}} / R_{\mathrm{i}}$ is the ratio of the load resistance to the internal resistance. Clearly, an $I-V$ curve described by Eq. 1 can be obtained by performing a fast scan, whilst an $I-V$ curve described by Eq. 2 can be obtained by performing a very slow scan. It has been shown theoretically ${ }^{8}$ that the short circuit current for a thermoelectric module operating under a constant temperature difference, $I_{\Delta}$, can be determined by the intercept with the current axis from the $I-V$ curve of Eq. 1. The short-circuit current for a thermoelectric module operating with a given thermal input, $I_{\mathrm{Q}}$, can be determined by the intercept with the current axis from the $I-V$ curve of Eq. 2. Consequently, the effective $Z T$ of thermoelectric modules can be calculated using ${ }^{8}$ :

$$
Z \bar{T}=\frac{I_{\Delta}}{I_{\mathrm{Q}}}-1
$$

In addition, the maximum power output and conversion efficiency of a thermoelectric module operating for a given thermal input can be calculated using ${ }^{8}$ :

$$
\begin{gathered}
P_{\max }=\frac{1}{4} I_{\mathrm{Q}} \cdot V_{\mathrm{o}} \\
\eta=\frac{1}{4} \frac{\Delta T_{o}}{\bar{T}}\left(1-\frac{I_{Q}}{I_{\Delta}}\right)
\end{gathered}
$$

Equations 5 and 6 provide a convenient method to characterize the performance of thermoelectric modules operating under a given thermal input. Currently, there is no clearly defined measurement technique for evaluation of thermoelectric modules under a given thermal input. This method has potential to provide a standard evaluation technique in this aspect.

\section{EXPERIMENTAL SETUP}

Figure 2a shows a schematic diagram of the measurement system, where the thermoelectric module is sandwiched between a heater and a cooling plate. To ensure minimum heat loss from the heater to the surrounding atmosphere, a heater that has the same area as that of the module is employed. The sandwiched part is placed in a vacuum chamber and covered with a heat shield to

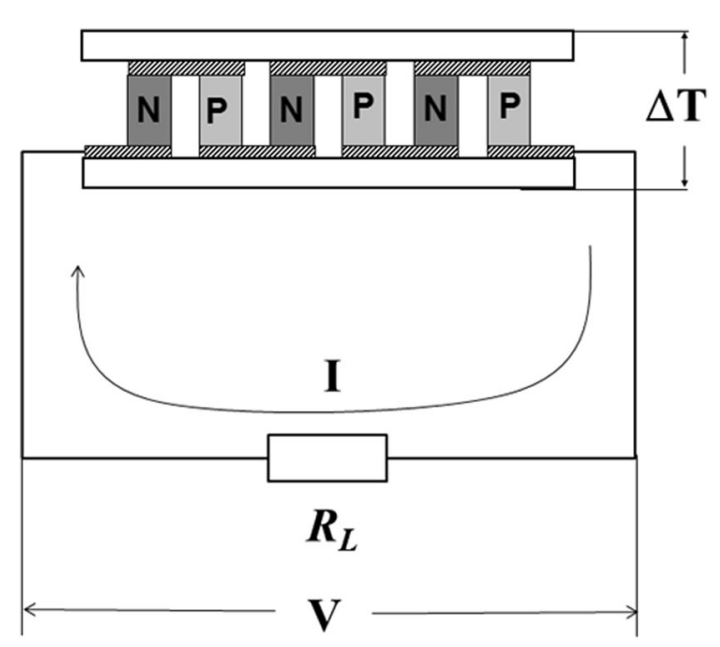

Fig. 1. Schematic diagram for measuring $I-V$ of thermoelectric module. 
minimise the heat losses due to convection and radiation, respectively. Measurements can be performed in vacuum or inert atmosphere, which enables high temperature characterisation up to $900 \mathrm{~K}$. Figure $2 \mathrm{~b}$ shows a photograph of the vacuum chamber of the system.

An important piece of equipment for satisfying the key requirements of the principle of measurement is essentially a potentiostat (Autolab PGSTAT302N) supplied by Metrohm Autolab, which can be directly connected to the outputs of a thermoelectric module and obtain a full $I-V$ curves at a controllable scan rate in a range of seconds to hours. This is the key to enable the $I-V$ measurement to be performed at constant temperature difference or constant thermal input. Since a thermoelectric module usually has a large thermal mass, the change in its hot side temperature lags significantly behind the action when the module switches from open-circuit to closed-circuit. It usually takes a few seconds before a reduction in temperature difference can be observed. Consequently, a fast scan (typically $<1 \mathrm{~s}$ ) will provide an $I-V$ trace, which corresponds to a constant temperature difference. On the other hand, the equipment can be programmed to take the $I-V$ data after a relatively long period (typically $1-2 \mathrm{~h}$ in our experiment) once the module is changed to a new setvalue of load resistance while keeping the input heat power constant. This corresponds to the measurement for a constant thermal input.
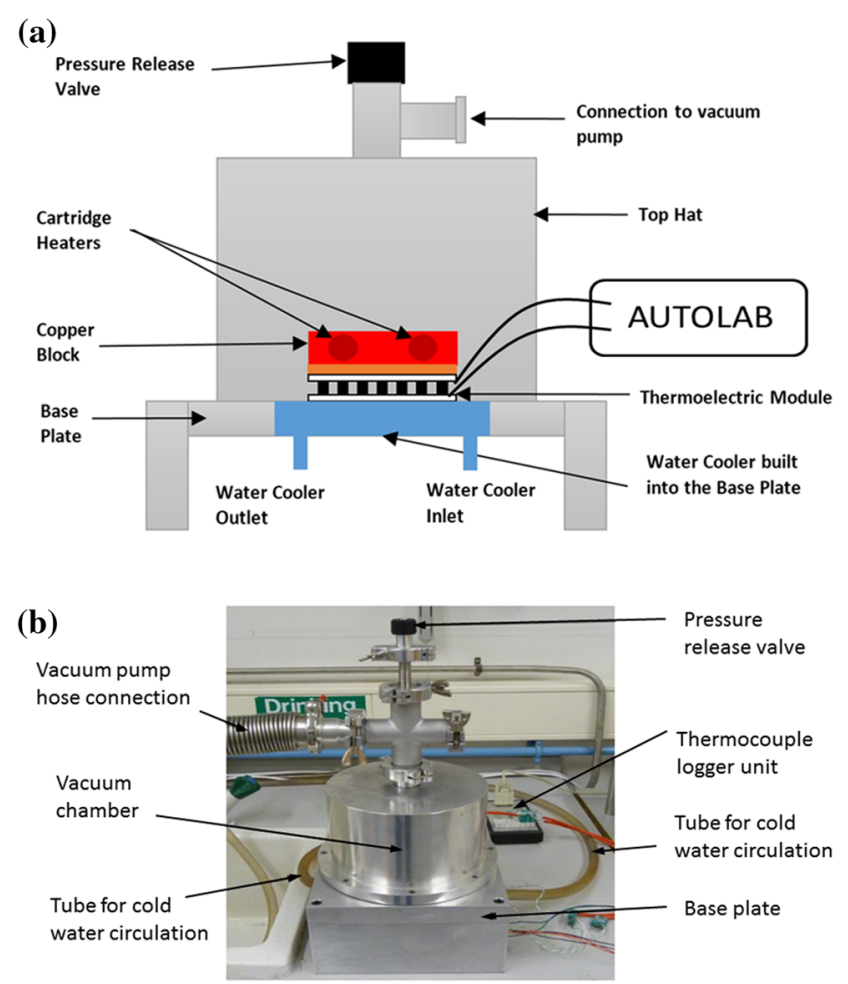

Fig. 2. Thermoelectric $I-V$ measurement system. (a) a schematic diagram; (b) the vacuum chamber.
In addition, the same Autolab system can provide a.c. excitation at a wide range of frequencies to a thermoelectric module. Under such an operating condition, a different technique (i.e. the impedance spectroscopy) can be employed to determine the $Z T$ values. ${ }^{9-11}$ In this work, we use the data from the impedance spectroscopy technique as a reference to validate the proposed technique. The impedance measurements were performed in a separate vacuum setup specifically designed for thermoelectric characterisation. A band heater with a diameter of $60 \mathrm{~mm}$ and a height of $65 \mathrm{~mm}$ was mounted inside the vacuum chamber. The thermoelectric module was lowered into the central region of the band heater and suspended from the top by its electrical leads. The band heater provides the ambient temperatures for the thermoelectric module through thermal radiation. The impedance measurements were carried out using an a.c. input current of $20 \mathrm{~mA}$ and frequencies of $0.01 \mathrm{~Hz}$ to $10 \mathrm{kHz}$.

\section{RESULTS AND DISCUSSIONS}

The thermoelectric module employed for this experiment was obtained from a commercial module manufacturer. It has 127 thermocouples and a surface area of $40 \mathrm{~mm} \times 40 \mathrm{~mm}$. The module was mounted in the measurement chamber as shown in Fig. 2 with a vacuum pressure of about $10^{-5}$ Torr. At open circuit, a temperature difference of $20 \mathrm{~K}$ was initially established across the thermoelectric module. An $I-V$ curve was obtained with a fast scan rate of about $0.5 \mathrm{~V} / \mathrm{s}$ using the Autolab system, and then another $I-V$ curve was obtained by taking data at a slow rate so that the voltage is measured only when the thermoelectric module has reached a steady state after changing the load resistance. Figure 3 shows the $I-V$ curves obtained for a constant temperature difference (solid circles) and that for a constant thermal input (solid squares). It can be seen that the experimental data confirms the linear relationships for both operating conditions with the small slope for the case when operating under a constant thermal input. Furthermore, the intercepts can be determined from the $I-V$ curves, which give $I_{\Delta}=0.36 \mathrm{~A}$ and $I_{Q}=0.21 \mathrm{~A}$, respectively. Using Eq. 4, the effective $Z T$ of thermoelectric module is 0.71 . It can be seen below that this is in a good agreement with the value obtained from the impedance spectroscopy technique, providing experimental verification of the proposed technique.

Figure 4 shows the corresponding power output of the thermoelectric module under a constant temperature difference and a constant thermal input, respectively. It can be seen that the maximum power output obtained under a constant temperature difference is much larger than that obtained under a constant thermal input. This is because the input thermal power to the module was increased with decreasing the load resistance in order to maintain a constant temperature. On the other 


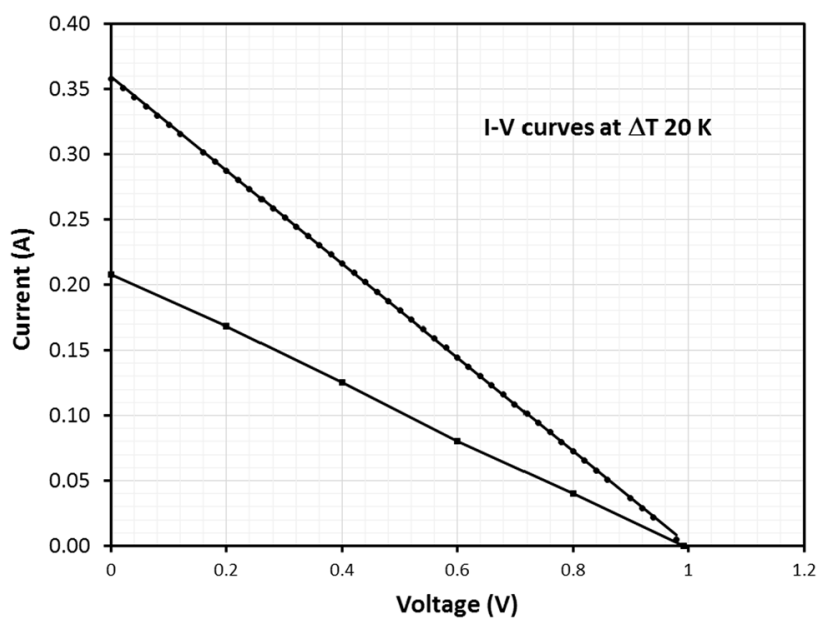

Fig. 3. $I-V$ curves obtained under a constant temperature difference (solid circles) and under a constant thermal input (solid squares), respectively.

hand, the temperature difference across the module will decrease with decreasing load resistance when the thermal input power is maintained constant. Furthermore, it can be seen that the optimal load resistance for operation under a constant temperature difference is very similar to the internal resistance (so-called matched resistance as expected). However, when thermal input power is maintained, the optimal load resistance for obtaining maximum power output is achieved at a larger resistance value $\left(\sim 1.8 R_{\mathrm{i}}\right)$. This is a result of the trade-off between approaching the matched load for maximum power output and increasing the load resistance to obtain a larger temperature difference across the thermoelectric module. Clearly, this result demonstrates that obtaining the optimal condition for maximum power output differs significantly depending on whether the module is operated in the constant temperature difference or the constant thermal input regime. This technique (obtaining the $I-V$ curve with a slow rate) provides an effective and convenient approach to determine the maximum power output and corresponding optimal load resistance. Clearly, the measurement accuracy depends strongly on the ability to maintain a constant thermal input during the slow scan. It is estimated that the measures employed to minimise the heat losses described in the previous section are sufficient to ensure the thermal input power is maintained constant within 5\% over the temperature ranges investigated. However, the error could increase if the temperature difference is much larger. In this case, a more sophisticated control and monitor system is needed.

A further experiment was carried out to demonstrate the capability of determining $Z T$ under any temperature difference. As discussed in, ${ }^{6}$ the thermoelectric figure-of-merit determined using the conventional technique is for a relatively small

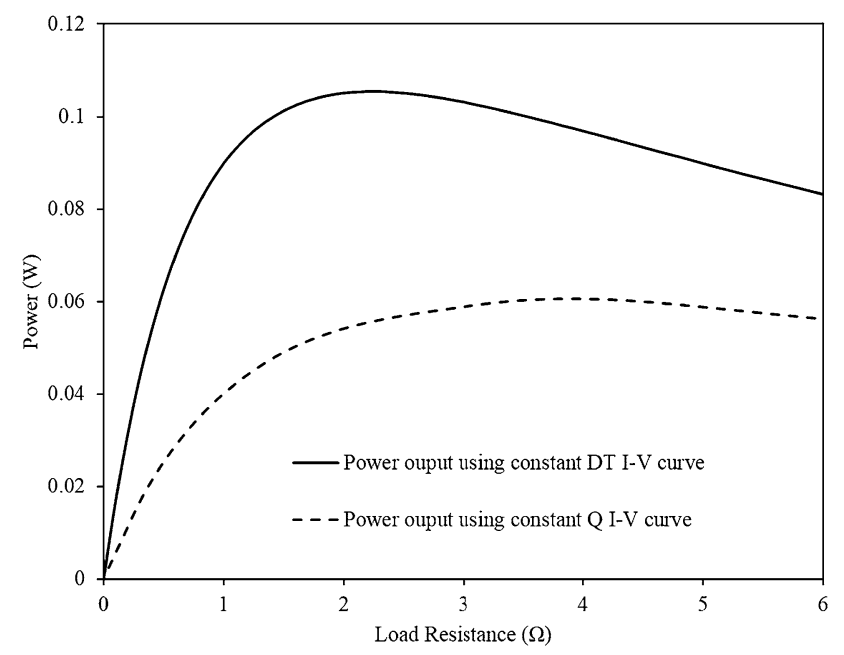

Fig. 4. Power outputs of the thermoelectric module as a function of the load resistance. The solid circles represent the power output obtained under a constant temperature difference $(20 \mathrm{~K})$ and the solid squares represent that obtained under a constant thermal input.

temperature difference $(10 \mathrm{~K}-20 \mathrm{~K})$, whilst most thermoelectric generators are usually operated under a much larger temperature difference $(>50 \mathrm{~K})$. Determination of $Z T$ under a large temperature difference provides useful insights into the realistic performance of thermoelectric generators (when operating under large temperature differences) and has the potential to detect the contributions of mechanisms, which are only significant under large temperature differences, such as the Thomson effect. Figure 5 shows the three sets of $Z T$ values as a function of the mean temperature $\left(\left[T_{\mathrm{H}}+T_{\mathrm{C}}\right] / 2\right)$, which were measured (1) by impedance spectroscopy in suspended conditions in vacuum, (2) by $I-V$ curves with $\Delta T$ maintained around $10 \mathrm{~K}$, and (3) by $I-V$ curves with increased $\Delta T$ up to $75 \mathrm{~K} . Z T$ measurement by impedance spectroscopy is in fact an improved Harman method. The temperature difference across the thermoelectric module is usually less than $1 \mathrm{~K}$ and does not require thermal contacts, so it is expected that the $Z T$ obtained should be slightly larger than from the $I-V$ technique, which involves formation of thermal contacts. Nevertheless, it can be seen that the results obtained from the $I-V$ technique under a small temperature difference are in good agreement with those obtained by impedance spectroscopy. This result demonstrates the validity of the proposed technique. Furthermore, it can be seen that the effective $Z T$ obtained under a large temperature difference for a given mean temperature is significantly smaller than that obtained under a small temperature difference, indicating that the real performance of the thermoelectric module will be much poorer than what is predicted from $Z T$ values under a small $\Delta T$ measurements. This result demonstrates the value of the $Z T$ measurement under a large temperature difference. 


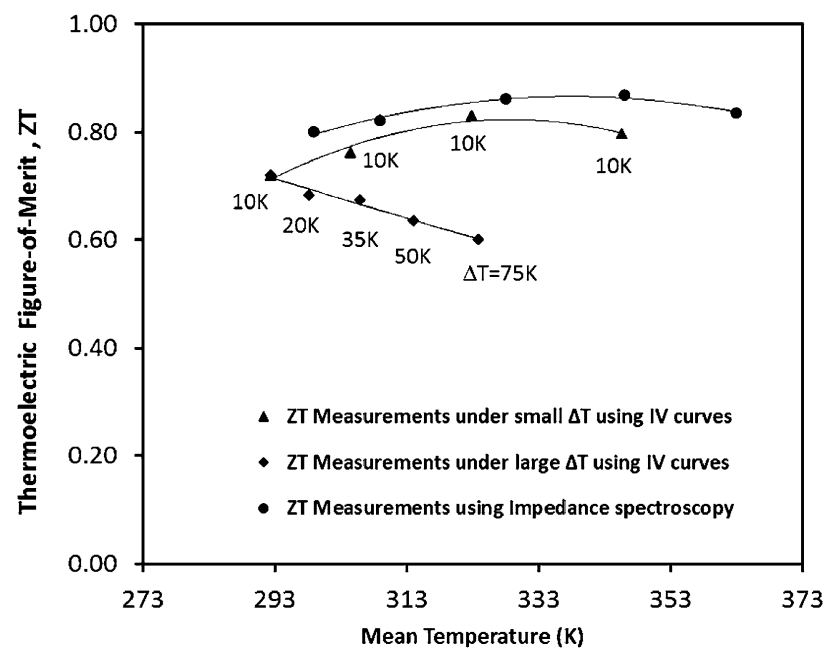

Fig. 5. $Z T$ values as a function of the mean temperature of the thermoelectric module. The solid circles represent $Z T$ values obtained by impedance spectroscopy; the solid triangles represent $Z T$ values measured by $I-V$ curves with $\Delta T$ maintained around $10 \mathrm{~K}$; and the solid diamonds represent $Z T$ values determined from $I-V$ curves with increased $\Delta T$ up to $75 \mathrm{~K}$.

It is to be noted that the conversion efficiency of the thermoelectric module can be determined using Eq. 6. However, in this work, we only focus on investigating the $Z T$ s. This is because the reference data can be readily obtained using the impedance spectroscopy technique, whilst obtaining reliable reference data for the conversion efficiency is more challenging.

\section{CONCLUSIONS}

A measurement system for thermoelectric characterisation based on $I-V$ curves has been developed which enables the $I-V$ curves of thermoelectric modules to be obtained at a controllable scan rate from less than a second to a few hours. The experimental results obtained using this equipment confirms the validity of a recently proposed thermoelectric measurement technique. ${ }^{8}$ This novel technique provides an effective method to determine the maximum power output and optimal load resistance of thermoelectric generators operating under a constant thermal input. The technique can also be used to determine the effective $Z T$ of thermoelectric devices with a capability of $Z T$ measurement under large temperature differences. This has important implications in understanding the realistic performances of thermoelectric generators in actual operating conditions. The results from a commercial module show that the $Z T$ obtained under a large temperature difference is significantly smaller than that under a small temperature difference.

\section{ACKNOWLEDGEMENTS}

The work is financially supported by the European Commission Research Fund for Coal and Steel programme under the Therelexpro project (RPS-PR12068) and Globasol project (309194).

\section{OPEN ACCESS}

This article is distributed under the terms of the Creative Commons Attribution 4.0 International License (http://creativecommons.org/licenses/by/4.0/), which permits unrestricted use, distribution, and reproduction in any medium, provided you give appropriate credit to the original author(s) and the source, provide a link to the Creative Commons license, and indicate if changes were made.

\section{REFERENCES}

1. A.F. Ioffe, Semiconductor Thermoelements and Thermoelectric Cooling (London: Infosearch, 1957), p. 37.

2. D.M. Rowe and C.M. Bhandari, Modern Thermoelectrics (London: Holt, Rinehart and Winston, 1983), p. 24.

3. D.M. Rowe, CRC Handbook of Thermoelectrics (London: CRC Press, 1996), p. 479.

4. S. Beeby and N. White, Energy Harvesting for Autonomous Systems (Norwood: Artech House, 2010), p. 135.

5. G. Min and NMd Yatim, J. Phys. D Appl. Phys. 41, 222001 (2008).

6. T.C. Harman, J. Appl. Phys. 29, 1373 (1958).

7. G. Min, J. Electro. Mater. 39, 1782 (2010).

8. G. Min, Meas. Sci. Technol. 25, 085009 (2014).

9. A.D. Downey, T.P. Hogan, and B. Cook, Rev. Sci. Instrum. 78, 93904 (2007).

10. A. De Marchi and V. Giaretto, Rev. Sci. Instrum. 82, 104904 (2011).

11. J. García-Cañadas and G. Min, J. Appl. Phys. 116, 174510 (2014). 